RUNNING HEAD: Attempts to control pain

ATTEMPTS TO CONTROL PAIN PRIORITIZE ATTENTION TO SIGNALS OF PAIN:

AN EXPERIMENAL STUDY

Lies Notebaert, Geert Crombez, Julia Vogt, Jan De Houwer and Stefaan Van

Damme,

Ghent University, Belgium

Jan Theeuwes

Vrije Universiteit, Amsterdam, the Netherlands 


\begin{abstract}
Clinical evidence suggests that a persistent search for solutions for chronic pain may bring along costs at a cognitive, affective and behavioural level. According to one hypothesis, attempts to control pain fuel hypervigilance and prioritize attention towards pain-related information. This hypothesis was investigated in an experiment with 41 healthy volunteers. Prioritization of attention towards a signal for pain was measured using an adaptation of the visual search paradigm. A previously neutral stimulus (Conditioned Stimulus, CS+) became a signal for pain (Unconditioned Stimulus: US, electrocutaneous stimulus at tolerance level) using a classical conditioning procedure. Intermixed with the visual search task participants also performed another task. In the pain control group, participants were informed that correct and fast responses on trials of this second task would result in an avoidance of the US. In the comparison group, the performance of the second task was not instrumental in controlling pain. Results showed that in the pain control group attention was more prioritized towards the CS+ than in the comparison group. Results are discussed in terms of a neuropsychological model of attention to pain.
\end{abstract}

Keywords: pain, attentional bias, avoidance, fear, anxiety, hypervigilance, control 


\section{ATTEMPTS TO CONTROL PAIN PRIORITIZE ATTENTION TO SIGNALS OF PAIN: AN EXPERIMENAL STUDY}

Imagine a man suffering from persistent back pain and worrying about the future. He considers possible ways out, visits numerous doctors, and tries several body postures to keep pain under control. Will he become hypervigilant to pain and pain-related information?

Pain demands attention [Eccleston \& Crombez, 1999] and often interferes with the accomplishment of valued activities [KarolyRuehlman2007]. It will be no surprise that, initially, individuals seek pain relief and attempt to get rid of the pain. Indeed, trying to control pain is a typical first strategy. When the efforts to seek pain relief are not immediately successful, individuals generally do not give in quickly, but simply increase the effort they put in their quest [VanDamme2008toprev]. Persistent attempts to solve the pain problem may however prove futile and even harmful when, like with chronic pain, the pain is a largely insoluble problem.

Recent theory suggests that the tenacious pursuit of pain relief may bring along costs at a cognitive, affective and behavioral level [AldrichBRAT; EcclestonCrombez2009; McCracken2007]. Ironically, attempts to control pain may fuel hypervigilance to pain and lead to an automatic capture of attention by painrelated information [EcclestonCrombez2009]. To date, this argument is largely based on clinical studies using largely self-report methods. For example, persistent attempts to solve chronic pain, as measured by the Pain Solutions Questionnaire, are related to self-reports of heightened attention to pain and affective distress [DeVlieger2006]. Conversely, the willingness to experience pain without the need to control, as measured by the Chronic Pain Acceptance Questionnaire [McGuire, in press], is 
positively related to cognitive, social and physical functioning, and negatively related to attention to pain [McCracken2007; Vianeetal2004]. To our knowledge, there is only one experimental study by Crombez et al (2008) that revealed that attempts to remain in control of pain came along with attentional costs. However, in that study it was not possible to identify the origins of the attentional costs, as only a crude measure of attention was used (task decrement on a secondary task).

The objective of this study was to investigate whether attempts to control pain lead to an attentional bias, and more specifically to an automatic capture of signals of pain. We used an adaptation of a visual search paradigm that allows investigating attentional bias to learned signals of pain via classical conditioning. In this paradigm, participants identify a target (line segment) presented in one of a variable number of colored circles, of which one (the CS+) is a signal for pain (see Figure 1). Overall, the time to find the target increases with the number of circles presented, rendering a steep search slope. Previously, we found that when the target was presented in CS+, the search slope was attenuated, but not completely flat [Notebaert et al., submitted]. This indicates that attention is prioritized, but not captured by the CS+. Of particular interest to the current study was whether attention is automatically captured by the CS+ when participants attempt to control pain.

\section{Method}

\section{Participants}

Forty-five students of Ghent University participated in partial fulfillment of course credit. After the acquisition phase, four participants were not able to correctly identify the pain-related color, and were removed from further analyses. The final 
sample of consisted of 22 participants in the pain control group (11 males, mean age $=18.9$ years, $S D=2.1)$ and 19 in the comparison group ( 6 males, mean age $=18.4$ years, $S D=1.0)$. All had normal or corrected-to-normal vision, and reported not to be color-blind. Participants gave their informed consent, and were informed that they could terminate the experiment at any time. No one made use of this option. The study was approved by the university's ethics committee.

\section{Stimulus Material and Apparatus}

The experiment was programmed using the E-Prime software package [Schneideretal2002]. Participants were seated approximately $60 \mathrm{~cm}$ from the screen.

A graphical representation of the stimuli in the visual search task can be found in Figure 1. On each visual search trial, the computer display consisted of a varying number of circles $\left(2.9^{\circ}\right.$ diameter $)$ with a colored band $\left(0.5^{\circ}\right.$ and black outlined $)$ against a silver background color. These colored circles were spaced equally distant from the midpoint of an imaginary circle (radius of $6^{\circ}$ visual angle, center of the screen). The number of stimuli presented (three, five or seven) is the set size. All circles in the display had different colors. There were nine possible colors: pink, blue, turquoise, yellow, green, orange, purple, red and grey. These colors were matched for intensity and luminance. The colored circles in the display contained a black line segment (extending $1^{\circ}$ ) in their centre. All line segments, except one, were tilted $\left(22.5^{\circ}\right.$ to either side of the horizontal or vertical plane; adopted from [Theeuwes1991]). The other line segment was the target stimulus, and was either a horizontal or a vertical line segment. 
On each action trial the display consisted of one colored circle that, in contrast to the visual search trials, was presented at the center of the computer screen. Other perceptual features were identical to those from a visual search trial.

Electrocutaneous stimuli were delivered by a constant current stimulator (DIGITIMER, model DS7A), and administered to the inside of the wrist of the nondominant forearm by two lubricated Fukuda standard $\mathrm{Ag} / \mathrm{AgCl}$ electrodes $(1 \mathrm{~cm}$ diameter). The electrocutaneous stimuli consisted of a series of 38 rectangular pulses (2 ms in duration with an inter pulse interval of $6 \mathrm{~ms}$ ), and had a total duration of $300 \mathrm{~ms}$. The intensity of the US was the maximum intensity that participants were willing to tolerate.

\section{Procedure}

Upon arrival, the tolerance level of the electrocutaneous stimulus was determined. To increase the threat value of the electrocutaneous stimulus, participants were fictitiously informed that even though most of the electrocutaneous stimuli would be of an intensity at their tolerance level, the intensity could be increased during the experiment [Crombezetal1998].

Next, participants practiced the visual search task with 15 trials. Electrocutaneous stimuli were not applied during the practice phase. During the subsequent acquisition phase, participants were instructed to find out which color (Conditioned Stimulus, CS+; counterbalanced across participants) was linked to the electrocutaneous stimulus (Unconditioned Stimulus, US). Colored circles without line segments were presented. To facilitate acquisition, this phase started with eight trials of set size 1 , followed successively by four trials of set size 3,5 and 7 . On half of the trials, the CS+ was presented. Half of the CS+ trials were followed by the US (partial 
reinforcement schedule of $1 / 2$ ) which was presented after CS+ offset. At the end of the acquisition phase, participants had to report which color was linked to the US.

The experiment phase consisted of four blocks of 141 trials. In each block, there were 94 visual search trials and 47 identification trials, presented at a fixed sequence of 2:1 in order to facilitate switching between tasks (procedure adopted from Vogt et al., in press).

On visual search trials, there were three types of trials (see Figure 1). (1) During congruent trials, the target was presented in the CS+; (2) During incongruent trials, the CS+ was present but the target was depicted in another colored circle; (3) During baseline trials, a target, but no CS+ was present. In order for the pain-related color (CS+) to remain threatening and predictive of the US, we chose the following procedural aspects. First, only half of the trials contained CS+. Second, during each block, four trials in which the CS+ was followed by the US, were added to avoid extinction [Mackintosh1974]. To make sure that participants could not strategically use the CS+ to localize the target, we used the $1 / n$ procedure (where $n$ is set size) so for each set size the CS+ was not predictive of the target [Jonides\&Yantis1988]. This means that for set size 3 , one out of three CS+ trials was a congruent trial, and this was done similarly for set size 5 and 7 . A detailed account of the distribution of trials can be found in Table 1. Participants were instructed to focus on the fixation cross at the beginning of each trial. Each trial started with a fixation cross at the center of the screen for a duration of $1000 \mathrm{~ms}$ after which the stimulus display was presented until response. Error feedback was displayed for $500 \mathrm{~ms}$. The inter trial interval was 750 ms. The speed of target identification (whether the line segment was horizontal or vertical) was measured using a two button response box. Responses had to be made with the index and middle finger of the dominant hand. 
*** Table $1^{\star \star *}$

Identification trials started with a fixation asterix (1000 ms) after which the stimulus was presented for a duration of $1000 \mathrm{~ms}$ or until a response was made. Error feedback was given for $500 \mathrm{~ms}$. In the pain control group, participants were informed that they could avoid the delivery of the US on that trial by pressing the spacebar as quickly as possible with the non-dominant hand when the CS+ was presented. They were informed that a fast response would lead to a $90 \%$ probability to avoid pain on that trial, and that a slow response would lead to a $90 \%$ probability that pain would be delivered on that trial. This probability factor was included to raise the credibility of the instructions. Whenever another stimulus was presented, no response had to be made. In the comparison group, participants were instructed to press the spacebar as fast as possible when the CS+ was presented. No information was given over the putative instrumentality of these trials. In fact, in both the pain control group and the comparison group, response speed and accuracy did not influence US probability. All participants were administered an equal number of USs. Out of the 47 identification trials in each block, the CS+ was presented on seven trials, three of which were followed by a US (and the error feedback "Too slow" was given in the pain control group).

After acquisition and at the end of the experiment, participants were requested to report the how intense, painful and freighting the US was $(0=$ Not at all, to $9=$ Extremely) and how unpleasant $(0=$ Very unpleasant, to $5=$ Neutral, to $10=$ Very pleasant) using Likert scales. At the end of the experiment, in order to assess whether conditioning was successful, participants also reported to what extent the US was expected after presentation of the CS+ $(0=$ Never, to $9=A /$ ways $)$, and how fearful they were during the presentation of the CS+ $(0=$ Not at all, to $9=$ Very $)$. In 
addition, participants reported how much control they felt they had over the presentation of the US in the identification task and the visual search task $(0=$ No control, to $9=$ A lot of control).

\section{Results}

\section{Data Trimming}

Trials on which an US was presented were not taken into account for analyses. Also trials with response errors (6.6\%) and with outliers $(2.6 \%$, defined as reactions times that deviated more than three standard deviations from the individual mean of correct responses, calculated for every trial type and set size separately) were removed. We calculated Cohen's $d$ and its $95 \%$ confidence interval $(\mathrm{Cl})$ for relevant terms. For ease of comparison with the norms of Cohen (1988), we calculated effect sizes for dependent samples using the formula of Morris and DeShon (2002) [see Borensteinet al2009]. An effect size of approximately 0.20 is considered a small effect, around 0.50 a medium effect and 0.80 to infinity a large effect [Cohen1988]. Standard deviations of the means are reported between brackets.

Descriptive statistics and manipulation check

Participants rated the US as rather unpleasant $(M=4.3[2.0])$, frightening $(M=$ 5.5 [2.1]), intense $(M=5.8$ [1.3]), and painful ( $M=4.4$ [1.9]). They reported to be afraid when the $C S+$ was presented on screen $(M=4.8$ [2.7]) and quite often expected a US to follow ( $M=6.1$ [2.2]). There were no differences between groups 
(all $p>.09$ ) or -regarding the US ratings- between measurement moments (all $p>$ $.07)$.

Self reports regarding the feeling of control over the presentation of the US for the identification trials did not differ between the pain control group $(M=4.1$ [2.7]) and the comparison group $(M=3.7[2.9] ; t<1)$. The same was true for self reports concerning control over the US in the visual search task (pain control group: $M=3.6$ [3.2]; comparison group: $M=3.1$ [2.5]; $t<1$ ). The pain control group was significantly faster $(M=418$ [121] $\mathrm{ms}$ on identification trials than the comparison group $(M=654$ [231]; $t(39)=3.99, p<.001)$. In addition, the pain control group had a lower accuracy on identification trials $(M=97.8[2.4] \%)$ than the comparison group $(M=$ $99.3[1.1] \% ; t(39)=2.49, p<.05)$. Inspection of the error pattern revealed that the pain control group made more false alarms $(87.2 \%)$ than misses $(12.8 \%)$. In the comparison group there were $44.0 \%$ false alarms and $56.0 \%$ misses. Participants in the pain control group also were significantly faster $(M=418$ [121] $\mathrm{ms}$ on identification trials than participants in the comparison group $(M=654$ [231]; $t(39)=$ $3.99, p<.001)$.

\section{Visual Search Data}

In a first step, we explored whether our procedure resulted in an attention bias towards signals of pain. By subtracting congruent from incongruent trials, measured across set sizes, we can see that both groups show a large attentional bias effect: $M_{\text {diff }}=434$ [203] $\mathrm{ms}$ in the pain control group $(t(21)=10.01, p<.001, d=1.44, \mathrm{Cl}$ : $1.04-1.84)$ and $M_{\text {diff }}=358$ [113] $\mathrm{ms}$ in the comparison group $(t(19)=13.78, p<$ $.001, d=1.14, \mathrm{Cl}: 0.93-1.34)$. Analyses further revealed that both groups showed difficulties to disengage from the CS+, as indexed by slower reaction times on 
incongruent trials than on baseline trials: 107.7 [91.9] $\mathrm{ms}$ in the pain control group $(t(21)=5.50, p<.001 ; d=0.25, \mathrm{Cl}: 0.16-0.34)$ and 103.1 [59.0] $\mathrm{ms}$ in the comparison group $(t(18)=7.62, p<.001 ; d=0.36, \mathrm{Cl}: 0.26-0.45)$. There was no difference in interference between groups. $(t<1)$. Analyses further revealed that both groups showed facilitated engagement with the CS+, as indexed by faster reaction times on congruent trials than on baseline trials: 326.0 [153.4] $\mathrm{ms}$ in the pain control group $(t(21)=9.96, p<.001 ; d=1.35, \mathrm{Cl}: 0.98-1.72)$ and $255.1[110.6] \mathrm{ms}$ in the comparison group $(t(18)=10.1, p<.001 ; d=0.81, \mathrm{Cl}: 0.63-0.99)$. There was no difference in facilitation between groups $(t(39)=1.71, p=.095)$.

Next, we calculated the search slopes. A search slope is calculated by subtracting reaction times on set size 3 from those on set size 7 , and dividing it by 4 [Wolfe1998]. The resulting number is the mean increase in reaction time per additional item in the display. The slope on congruent trials was 51 [60] ms in the pain control group, and 93 [62] $\mathrm{ms}$ in the comparison group. Since the reaction time increase on congruent trials in both groups was above the criterion of $15 \mathrm{~ms}$ per additional item in the display [Wolfe1998], we may conclude that there is no evidence for attentional capture by threat. To investigate whether the CS+ is prioritized over the other stimuli [Frischenetal2009], we compared slopes on congruent and baseline trials. The pain control group showed a prioritization effect since the slope on congruent trials was significantly flatter than the slope on baseline trials (134 [38] ms; $t(21)=6.48, p<.001 ; d=1.61, \mathrm{Cl}: 0.88-2.34)$. Also in the comparison group, the slope on congruent trials was significantly flatter than the slope on baseline trials (151 [44] ms; $t(18)=5.46, p<.001 ; d=1.02, \mathrm{Cl}: 0.59-1.46)$.

Finally, and most importantly, we tested whether the attention towards the CS+ was more prioritized in the pain control group than in the comparison group. To 
this end, we compared the search slope on congruent trials between the pain control group and the comparison group. A planned comparison revealed that the slope on congruent trials was significantly flatter in the pain control group than in the comparison group $(t(39)=2.22, p<.05 ; d=.70, \mathrm{Cl}: 0.06-1.33)$. There was no difference between the two groups for the search slope on baseline trials $(t(39)=$ $1.34, p>.1$. A detailed account of these data can be viewed in table 2 .

*** Table 2 ***

\section{Discussion}

The present study investigated whether signals of pain automatically capture attention when participants attempt to control pain. Our results can be readily summarized. First, attention is biased to signals of pain, both in the pain control group and in the comparison group. Second, individuals who attempt to control pain show more prioritization of signals of pain than individuals who do not have this goal.

Even though attentional bias to threat is well established, the typical effects are usually moderate [BarHaim2007] to relatively small [Pincus\& Morley2001]. In our study, the effect size of our attentional bias to threatening information (reaction times on incongruent trials minus reaction times on congruent trials) is large. This may be due to procedural differences with other studies. First, whereas previous studies investigating attentional bias used complex stimuli (e.g., words, faces and pictures; [Roelofsetal2002EurJPain]), we used simple visual stimuli (e.g., colors) that may be especially effective in modifying the allocation of attention [LeDoux1998, Öhman\&Mineka2001]. Second, the classical conditioning procedure with the involvement of an actual threatening stimulus may have led to a situation that was 
threatening for all participants. Third, it may be that the selection of threatening information especially emerges in a context of multiple, competing stimuli [Crombezetal2005]. Indeed, in comparison with other paradigms such as the exogenous cueing (where only one stimulus is presented; [Van DammeLorenzetal2004]) and dot probe paradigm (where two stimuli are presented simultaneously; [Asmundsonetal2005], our visual search paradigm displays more information elements (set size). In line with previous studies of our lab, creating signals for pain using a classical conditioning procedure, results in both a facilitated engagement of attention to the CS+ and a difficulty to disengage attention from the CS+, once detected [VD06]. In contrast with previous findings [VanDammeetal], we here observed a strong engagement of attention with the CS+. Further results of our study indicate that a facilitation of engagement with the CS+, is best revealed in a context with several competing stimuli (set sizes).

Of particular importance was the finding that attempts to control pain increase prioritization of attention to signals of pain. This is an intriguing finding, as in fact, the signals of pain in the visual search task have no instrumental value in controlling pain. Indeed, only on identification trials, participants in the pain control group were informed that a fast response to the CS+ could result in avoidance of pain. Thus, it seems that installing a goal to control pain in one task, is sufficient to prioritize information that is congruent for that goal, even when this information is no longer instrumental for the task at hand. This view is in line with a recent neurocognitive model of attention and pain [Legrain2009]. According to this model, we select information that is relevant for current actions, based on our intentions and goals (top-down selection). The mental set of stimuli that are important for the current task is called the attentional set. Stimuli that share features with this attentional set are 
likely to capture attention [FolkRemingtonJohnston1992]. It may thus be expected that pain or signals of pain will capture attention when they are part of this attentional set. However, in our visual search task, the signal of pain is task-irrelevant (and therefore presumably not part of the attentional set) and thus, it may be expected that pain or the signal of pain will be ignored and not enter awareness. On the other hand, attention is involuntary captured by very salient stimuli in our environment, such as new, rare, or intense events (bottom-up selection). Because it is intrinsically threatening, pain is an eminent candidate for bottom-up attentional capture [Legrain2009]. The brain structures involved in attention to pain are thought to be the midcingulate cortex (MCC) which is thought to play a role in orienting attention to salient and potentially threatening stimuli (bottom-up selection) and the dorsolateral prefrontal cortext (DLPFC) and the intraparietal sulcus (IPS), which are thought to be important structures for upholding goals and preventing interference (top-down slection) [Legrain2009]. If our visual search results would only be influenced by bottom-up attentional selection, we should have found a flat search slope on congruent trials. The fact that we find a slope attenuation, but not a flat search slope, indicates some top-down control over the attentional capture by signals of pain [cfr Crombez VanDammeEccleston2005].

Our finding extends previous ideas about the origins of hypervigilance and attentional bias to pain-related information. In many clinical models [Crombezetal2005; VlaeyenLinton2000] pain-related fear is considered the key process for an emergence of hypervigilance to pain-related information. Questionnaire studies [GoubertCrombezVanDamme2004, Crombez Ecclestonet 2004clinical journal of pain] and experimental studies [Van Dammeetal2006?] have corroborated this view. However, this study suggests that attempts to control or avoid 
pain further fuel this hypervigilance. Not only what patients fear about pain, but also their attempts to control or avoid pain, matter. It indicates that a persistent search for solutions for pain may come along with attentional costs in patients with chronic pain [EcclestonCrombez2007, McCracken2007]. An important avenue for further research will be to further investigate the attentional dynamics towards pain and pain-related information in a context of multiple goals and motivations [VD2010].

The current study has some clinical implications. First, the adoption of a goal perspective may prove useful in identifying how and when pain can be ignored and does not easily enter awareness [VD2010]. Second, although we focused upon attentional costs in this study, we do not argue for a slavish use of attentional control strategies to target these costs. We have shown that attentional bias to signals of pain can be acquired by a classical conditioning procedure [ref]. It may, likewise, diminish by using an extinction or exposure procedure [VanDammeCrombezHermansetal2006]. Third, treatments that focus upon accepting pain and giving up attempts to control uncontrollable pain may be well suited in reducing a hypervigilance to pain and in improving daily functioning despite pain [McCracken2007; Vianeetal2004].

The finding that participants in the pain control group made more false alarms on identification trials than participants in the comparison group is compatible with another experimental study [Brandtstädteretal2004] and can be explained by a "better safe than sorry" strategy [Smeetsetal2000]. Indeed, pressing the spacebar when no CS+ was presented had no adverse consequences, whereas missing a CS+ presentation and receiving a US is not that pleasant. Combined with the finding that pain control group was faster on identification trials than the comparison group, 
these data suggest that participants in the pain control group were indeed actively trying to avoid the US on identification trials.

It is remarkable that an enhanced attentional bias was found even though the pain control group did not report to feel more in control over the presentation of the US than the comparison group. This shows that the enhanced bias cannot be explained merely by a difference between the groups in the subjective feeling of control. In contrast, these ratings seem to indicate that specifically the intentions to avoid the electrocutaneous stimulus sufficient to produce a stronger bias to pain signals. The reason why the control ratings in the pain control group are relatively low, could be because on identification trials the CS+ was still followed by a US on three out of seven trials. This might have given participants the feeling that their attempts to avoid the US were not very successful. On the other hand, it is likely that participants in the comparison group started developing hypotheses or strategies about the experiment and ways to influence it, which resulted in non-zero scores on the control ratings.

Finally, some limitations to this study must be addressed. First, since we tested healthy volunteers, we have to be cautious when generalizing these results to clinical samples. Second, in this study we did not address to what extent the enhanced prioritization of emotional stimuli is unique to actions related to pain. It may well be that similar effects can be found when one would for example reward participants for correct and fast responses in the additional task. 


\section{References}

Allport, D. A. (1993). Attention and control. Have we been asking the wrong questions? A critical review of twenty-five years. In D. E. Meyer \& S. Kornblum (Eds), Attention and performance XIV: Synergies in experimental psychology, artificial intelligence, and cognitive neuroscience (pp. 183-218). Cambridge, MA: MIT Press.

Bar-Haim, Y., Lamy, D., Pergamin, L., Bakermans-Kranenburg, M. J., \& van IJzendoorn, M. H. (2007). Threat-related attentional bias in anxious and nonanxious individuals: A meta-analytic study. Psychological Bulletin, 133, 124.

Bekkering, H., \& Neggers, S.F.W. (2002). Visual search is modulated by action intentions. Psychological Science, 13, 370-374.

Brandtstädter, J., Voss, A., \& Rothermund, K. (2004). Perception of danger signals: The role of control. Experimental Psychology, 51, 24-32.

Craighero, L., Fadiga, L., Rizzolatti, G., Umiltà, C., A. (1999). Action for perception: a motor-visual attentional effect. Journal of Experimental Psychology: Human Perception and Performance, 25, 1673-1692.

Crombez, G., Eccleston, C., Baeyens, F., Eelen, P. (1998). When somatic information threatens, catastrophic thinking about pain enhances attentional interference. Pain, 75, 187-98.

Darwin, C. (1872). The expression of emotions in man and animals. New York: Philosophical Library. 
Eccleston, C., \& Crombez, G. (2007). Worry and chronic pain: A misdirected problem solving model. Pain, 132, 233-236.

Fagioli, S., Hommel, B., \& Schubotz, R. I. (2007). Intentional control of attention: action planning primes action-related stimulus dimensions. Psychological Research-Psychologische Forschung, 71, 22-29.

Flykt, A. (2005). Visual search with biological threat stimuli: Accuracy, reaction times, and heart rate changes. Emotion, 5(3), 349-353.

Flykt, A. (2006). Preparedness for action: Responding to the snake in the grass. American Journal of Psychology, 119(1), 29-43.

Frijda , N.H . (1986). The emotions. Cambridge, UK: Cambridge University Press.

Frischen, A., Eastwood, J. D., \& Smilek, D. (2008). Visual search for faces with emotional expressions. Psychological Bulletin, 134, 662-676.

Gerritsen, C., Frischen, A., Blake, A., Smilek, D., \& Eastwood, J. D. (2008). Visual search is not blind to emotion. Perception \& Psychophysics, 70(6), 1047-1059.

Hannus, A., Cornelissen, F. W., Lindemann, O., \& Bekkering, H. (2005). Selectionfor-action in visual search. Acta Psychologica, 118(1-2), 171-191.

Hermans, D. (1994). De Zelf-Beoordelings-Vragenlijst (ZBV) [The State-Trait Anxiety Inventory (STAI)], Gedragstherapie, 27, 145-148.

Horstmann, G. (2009). Visual search for schematic affective faces: Stability and variability of search slopes with different instances. Cognition \& Emotion, 23(2), 355-379. 
Jonides, J., \& Yantis, S. (1988). Uniqueness of Abrupt Visual Onset in Capturing Attention. Perception \& Psychophysics, 43(4), 346-354.

Keltner, D., \& Gross, J. J. (1999). Functional accounts of emotions. Cognition \& Emotion, 13, 467-480.

Koster, E. H. W., Crombez, G., Van Damme, S., Verschuere, B., \& De Houwer, J. (2004). Does imminent threat capture and hold attention? Emotion, 4(3), 312317.

Lang, P.J. (1995). The emotion probe. American Psychologist, 5, 372-385.

LeDoux, J. (1998). Fear and the brain: Where have we been, and where are we going? Biological Psychiatry, 44, 1229-1238.

Mackintosh, N.J. (1974). The Psychology of Animal Learning. New York: Academic Press.

Macleod, C., Mathews, A., \& Tata, P. (1986). Attentional bias in emotional disorders. Journal of Abnormal Psychology, 95, 15-20.

Müsseler, J., \& Hommel, B. (1997). Blindness to response-compatible stimuli. Journal of Experimental Psychology: Human Perception and Performance, 23, 861872.

Neumann, O. (1987). Beyond capacity: A functional view of attention. In: H. Heuer \& A. F. Sanders (Eds.) Perspectives on Perception and Action, Erlbaum, Hillsdale. 
Notebaert, L., Crombez, G., Van Damme, S., De Houwer, J., \& Theeuwes, J. (submitted). Signals of threat do not capture, but prioritize attention: a classical conditioning approach. Emotion

Öhman, A., Flykt, A., \& Esteves, F. (2001). Emotion drives attention: Detecting the snake in the grass. Journal of Experimental Psychology: General, 130(3), 466478.

Öhman, A., \& Mineka, S. (2001). Fears, phobias, and preparedness: Toward an evolved module of fear and fear learning. Psychological Review, 108, 483522.

Pincus T, Morley S. Cognitive processing bias in chronic pain: a review and integration. Psychol Bull (2001) 127:599-617

Rizzolatti, G., Riggio, L., \& Sheliga, B. M. (1994). Space and selective attention. In C. Umilta, \& M. Moscovitch (Eds.), Attention and performance (pp. 231-265). Cambridge: MIT Press.

Schneider, W., Eschman, A., \& Zuccolotto, A. (2002). E-Prime User's Guide. Pittsburgh: Psychology Software Tools Inc.

Spielberger, C. D., Gorsuch, R. L., Lushene, R., Vagg, P. R., \& Jacobs, G. A. (1983). Manual for the State-Trait Anxiety Inventory. Palo Alto, CA: Consulting Psychologists Press.

Theeuwes, J. (1991). Cross-Dimensional Perceptual Selectivity. Perception \& Psychophysics, 50(2), 184-193. 
Van Damme, S., Crombez, G., Hermans, D., Koster, E. H. W., \& Eccleston, C. (2006). The role of extinction and reinstatement in attentional bias to threat: A conditioning approach. Behaviour Research and Therapy, 44(11), 1555-1563.

Van Damme, S., Legrain, V., Vogt, J., \& Crombez, G. (in press). Keeping pain in mind: A motivational account of attention to pain. Neuroscience and Biobehavioral Reviews.

Van der Ploeg, H. M., Defares, P. B., \& Spielberger, C. D. (1980). Handleiding bij de Zelf Beoordelings Vragenlijst. [Manual for the Dutch adaptation of the STAI-Y]. Lisse: Swets \& Zeitlinger.

Viane, I., Crombez, G., Eccleston, C., Devulder, J., \& De Corte, W. (2004). Acceptance of the unpleasant reality of chronic pain: effects upon attention to pain and engagement with daily activities. Pain, 112(3), 282-288.

Vogt, J., De Houwer, J., Moors, A., \& Crombez, G. (2008). The influence of explicit and implicit goals on the allocation of spatial attention. International Journal of Psychology, 43(3-4), 341-341. 


\section{Author Note}

Lies Notebaert, Geert Crombez, Julia Vogt, Stefaan Van Damme, Department of Experimental-Clinical and Health Psychology, Ghent University. Jan Theeuwes, Department of Cognitive Psychology, Vrije Universiteit, Amsterdam.

This research was funded by grants B/07386/02 and BOF/GOA2006/001 of Ghent University.

Correspondence concerning this article should be addressed to Lies Notebaert, Department of Experimental-Clinical and Health Psychology, Ghent University, Henri Dunantlaan 2, 9000 Ghent, Belgium. Email:

Lies.Notebaert@UGent.be 
Table 1

Distribution of trials in each of the 4 blocks.

\begin{tabular}{lccc}
\cline { 2 - 4 } & Set size 3 & Set size 5 & Set size 7 \\
\hline Congruent & 3 & 3 & 3 \\
\hline Incongruent & 6 & 12 & 18 \\
\hline Baseline & 9 & 15 & 21
\end{tabular}


Table 2

Mean reaction times $(S D)$ for each set size in every trial type for both groups.

\begin{tabular}{lllll}
\hline Set size 3 & Set size 5 & Set size 7 & Total & Slope
\end{tabular}

Pain control group

\begin{tabular}{rrrrrr}
\hline Congruent & $898(188)$ & $985(249)$ & $1104(346)$ & $964(231)$ & $51(60)$ \\
\hline Baseline & $953(193)$ & $1234(232)$ & $1489(296)$ & $1299(248)$ & $134(38)$ \\
& & & & & \\
\hline Incongruent & $1076(229)$ & $1324(294)$ & $1560(364)$ & $1402(318)$ & $121(46)$ \\
\hline Comparison group & & & & & \\
\hline Congruent & $977(230)$ & $1145(357)$ & $1351(433)$ & $1133(320)$ & $94(61)$ \\
\hline Baseline & $1026(222)$ & $1299(236)$ & $1631(356)$ & $1394(286)$ & $151(44)$ \\
\hline Incongruent & $1153(259)$ & $1369(286)$ & $1679(306)$ & $1493(289)$ & $131(31)$ \\
\hline
\end{tabular}




\section{Figure Captions}

Figure 1: Schematic examples of the different stimulus displays (not to scale).

Spotted circles represent the CS+. Panel A: Congruent trial of set size 3 with horizontal target. Panel B: Incongruent trial of set size 5 with vertical target. Panel C: Baseline trial of set size 7 with vertical target.

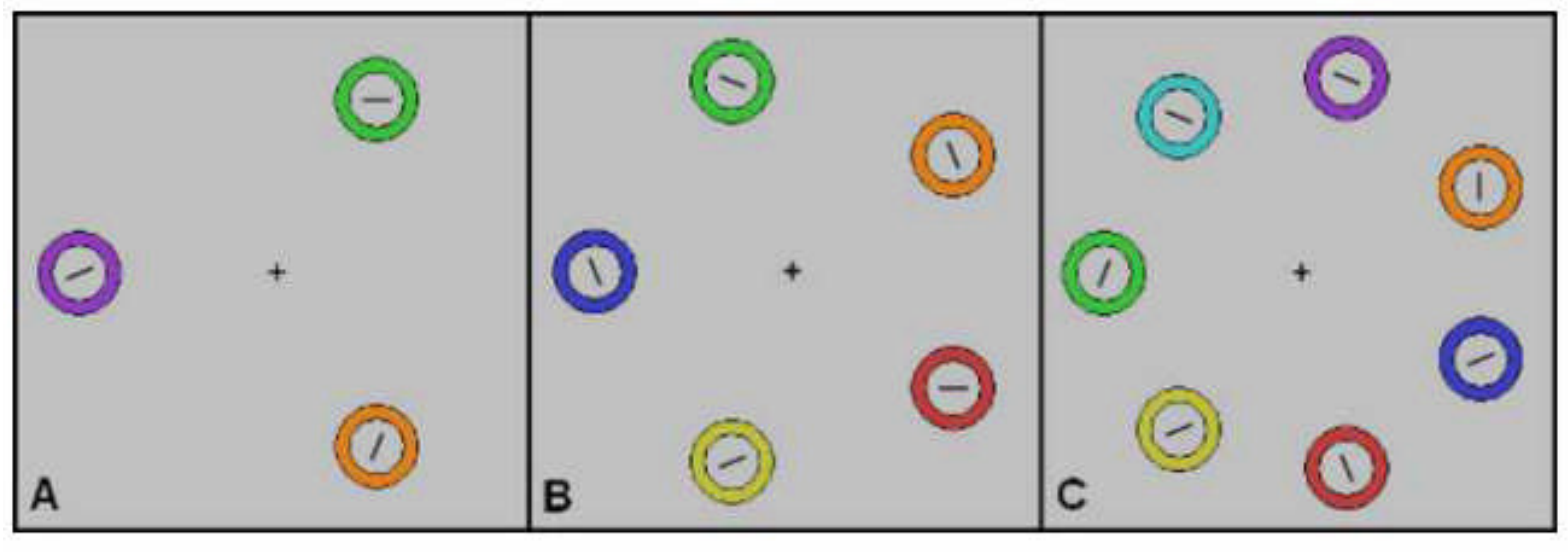


Figure 2: Mean reaction time and standard error lines for each set size of the congruent trials in the experimental and comparison group.

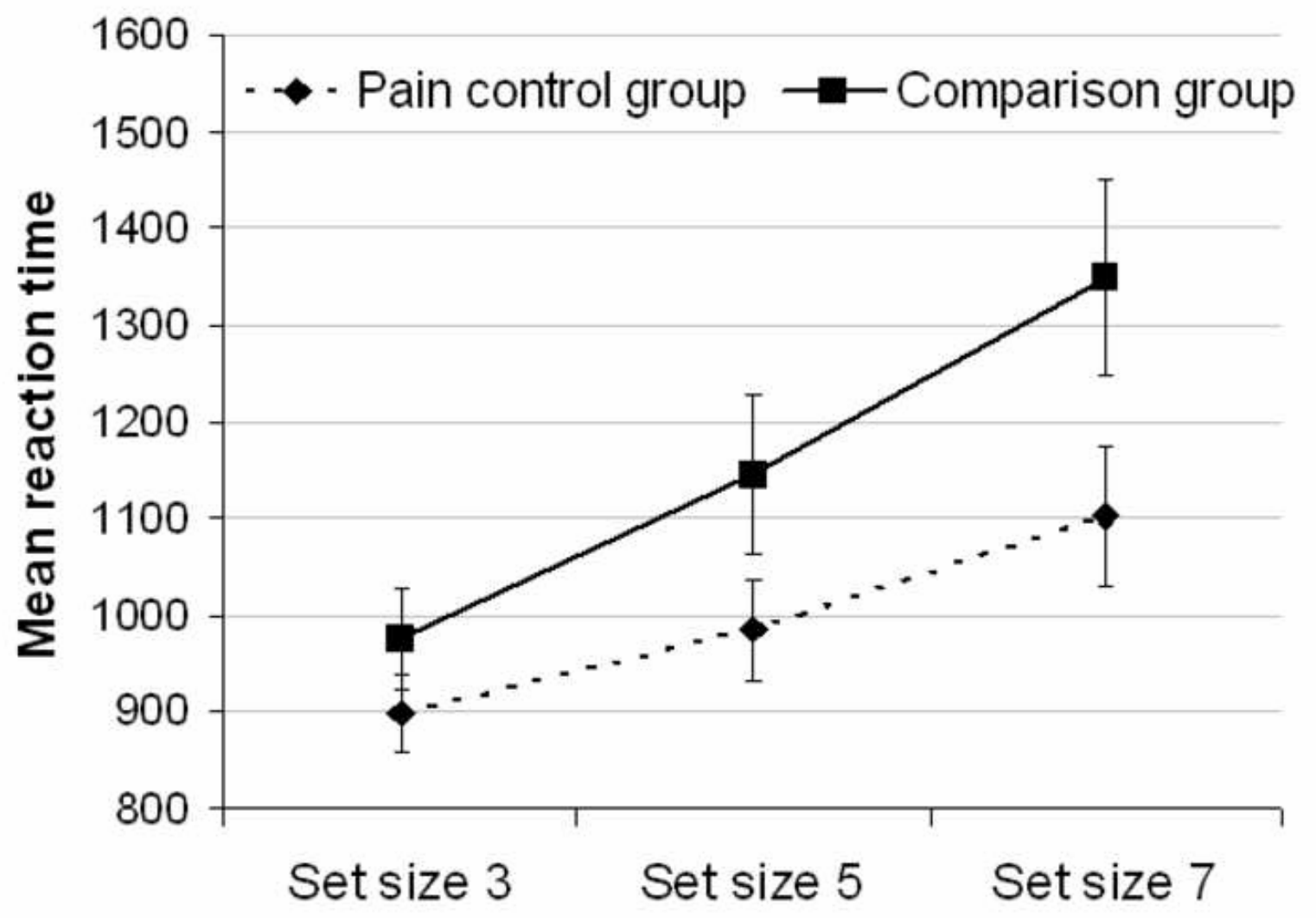

